\title{
Executive Functioning in Chinese Patients With Obsessive Compulsive Disorder
}

\begin{abstract}
Huicong Ren ${ }^{1 \dagger}$, Haibin $\mathrm{Li}^{2 \dagger}$, Jin Huang ${ }^{3+}$, Nan Zhang ${ }^{4}$, Ruiqin Chen ${ }^{5}$, Wenjuan Liu ${ }^{2 *}$, Zhaohui Zhang ${ }^{6 *}$ and Chencheng Zhang ${ }^{7,8 *}$

${ }^{1}$ The Second Department of Psychiatry, The Second Affiliated Hospital of Xinxiang Medical University, Xinxiang, China, ${ }^{2}$ Department of Psychological Medicine, Zhongshan Hospital, Fudan University, Shanghai, China, ${ }^{3}$ School of Public Health, Shanghai Jiao Tong University School of Medicine, Shanghai, China, ${ }^{4}$ Research Center of Brain and Cognitive Neuroscience, Liaoning Normal University, Dalian, China, ${ }^{5}$ Research Integrative Neuroscience, Deanery of Biomedical Sciences, University of Edinburgh, Edinburgh, United Kingdom, ${ }^{6}$ Departments of Psychiatry, The First Affiliated Hospital of Xinxiang Medical University, Xinxiang, China, ${ }^{7}$ Department of Neurosurgery, Center for Functional Neurosurgery, Ruijin Hospital, Shanghai Jiao Tong University School of Medicine, Shanghai, China, ${ }^{8}$ Shanghai Research Center for Brain Science and Brain-Inspired Intelligence, Shanghai, China
\end{abstract}

OPEN ACCESS

Edited by: Euripedes Constantino Miguel, University of São Paulo, Brazil

Reviewed by:

Marcelo Camargo Batistuzzo, Pontifical Catholic University of São

Paulo, Brazil

Nicole CR McLaughlin,

Butler Hospital, United States

*Correspondence: Wenjuan Liu

liu.wenjuan@zs-hospital.sh.cn Zhaohui Zhang

Zzhui816@126.com

Chencheng Zhang i@cczhang.org

†These authors have contributed equally to this work

Specialty section: This article was submitted to Mood and Anxiety Disorders, a section of the journal Frontiers in Psychiatry

Received: 01 February 2021 Accepted: 05 August 2021 Published: 25 August 2021

Citation: Ren H, Li H, Huang J, Zhang N, Chen R, Liu W, Zhang Z and Zhang $C$ (2021) Executive Functioning in Chinese Patients With Obsessive Compulsive Disorder.

Front. Psychiatry 12:662449. doi: 10.3389/fpsyt.2021.662449
Introduction: Studies have shown that patients with obsessive compulsive disorder (OCD) often perform more poorly than healthy control $(\mathrm{HC})$ participants on cognitive tasks involving executive functions. Most studies, however, have been performed in Western countries and societies, making it uncertain whether impaired executive functions can also be observed among non-Western patients with OCD. To address this gap in the literature, we evaluated several executive functions in Chinese patients with OCD and HCs.

Methods: Participants included consisted of 46 Chinese patients with OCD (25 men, 21 women), ranging in age from 19 to 56 years, and 45 matched HCs without any self-reported lifetime psychiatric disorder. They all lived in Shanghai or the surrounding area. Five tests of the Cambridge Neuropsychological Test Automated Battery (CANTAB) were used to evaluate several executive functions (response inhibition, spatial working memory, planning, and cognitive flexibility) along with testing basic learning and visual recognition memory. Statistical tests using a Bonferroni-corrected significance level of $p=0.003$ were performed to assess overall patient-control group differences in cognitive performance. Additionally, we explored performance differences between patients classified as having either relatively mild symptoms or severe symptoms based on the individual total scores on the Yale-Brown Obsessive-Compulsive Scale.

Results: There were no significant performance differences between patients with OCD and HC in any of the cognitive tests. Similarly, cognitive performance of patients with relatively mild OCD symptoms did not differ significantly from that of patients with severe symptoms.

Conclusions: These results do not seem to support the view that impaired executive functioning represents a basic cognitive and pathophysiological feature of Chinese patients with OCD. However, due to study limitations, additional research is required before this conclusion can be well accepted.

Keywords: obsessive-compulsive disorder, cognitive functions, CANTAB, executive function, yale-brown obsessive compulsive scale 


\section{INTRODUCTION}

Obsessive-compulsive disorder (OCD) is a psychiatric disorder characterized by recurrent obsessions and/or compulsions. Obsessions consist of intrusive repetitive thoughts, images, or impulses. Compulsions are purposeful, repetitive overt or covert behaviors or rituals that are performed by afflicted persons in an effort to relieve anxiety and distress (1). OCD has a lifetime prevalence of $1-3 \%$ and is equally common among women and men (2). Patients with OCD often experience a lower quality of life and impaired social and occupational functioning $(3,4)$.

Studies have shown that patients with OCD typically perform more poorly than matched healthy control participants (HCs) on neuropsychological tests involving high-level cognitive or 'executive' functions, including planning, working memory, cognitive flexibility, and inhibitory motor control (5-7). These results have led to the hypothesis that impaired executive functioning (EF), including hyper excitability of the orbital frontal cortex and its functional connections, is a core cognitive and pathophysiological feature of $\operatorname{OCD}(8,9)$. Indeed, the current dominant view on the neuropathology of OCD focuses on abnormalities in prefrontal-striatal circuits implicated in $\mathrm{EF}$ (10). EF refers to various general-purpose cognitive-control abilities, mainly supported by the prefrontal cortex (PFC), that allow individuals to regulate their thoughts and behaviors (11). EF deficits thus have important consequences for dailylife functioning and may be major contributors to the lack of cognitive flexibility and the perseverative, repetitive behaviors that are cardinal symptoms of OCD (12). Unfortunately, cognitive studies of patients with OCD have not always yielded consistent findings $(12,13)$. This makes it difficult to arrive at a clear picture of the cognitive functions that are impaired and those functions that are not impaired in patients with OCD. Gaining such understanding could help clinicians to target psychological interventions for OCD according to the integrity of the patients' cognitive functioning.

Meta-analytical reviews of the literature have identified various factors that probably contributed to the mixed findings of studies examining cognitive functioning in adult patients with OCD (12-14), One important factor is the type of cognitive task used to assess the patients' cognitive functioning, which varied greatly between studies (13). Another potentially important source of between-study variability in results is the size and nature of the patient sample examined, including clinical characteristics (e.g., symptom severity, medication status, presence of psychiatric comorbidities) and demographics (e.g., age, sex, intelligence). In this context, it should also be noted that the currently available data concerning cognitive function in OCD primarily come from Western countries and cultures, which raises the question whether the study results can be generalized to non-Western patient populations.

Against this background, the present study was designed to assess cognitive functioning in Chinese patients with OCD and HCs. Based on previous neuropsychological and neuroimaging studies of patients with OCD (8), we focused on several cognitive functions within the broad domain of executive functioning, particularly response inhibition, spatial working memory, planning, and cognitive flexibility. The study objective was twofold: (1) to evaluate several executive functions in Chinese patients with OCD and HCs along with testing their basic learning and memory, and (2) to evaluate differences in executive functions between patients classified as having either relatively mild or severe OCD symptoms. Our main hypothesis was that the patients with OCD, especially those with severe symptoms, would be characterized by impaired executive functions. To assess participants' cognitive functioning, we used several tests of the Cambridge Neuropsychological Test Automated Battery (CANTAB), which is a widely used, validated, and standardized neurocognitive test battery (15). The CANTAB is non-verbal in nature, non-sensitive to gender, and principally culture-free (16), which makes this instrument well-suited for the purpose of the present study.

\section{MATERIALS AND METHODS}

\section{Participants}

Forty-seven patients with OCD (diagnosed by an expert psychiatrist using clinical interview and WHO ICD-10 criteria) were recruited from the Department of Functional Neurosurgery of Ruijin Hospital and the Department of Psychological Medicine of Zhongshan Hospital over a 29month period (Jun 30, 2018-Nov 25, 2020). Forty-seven HCs were recruited from the community by means of local advertisements. Patients and HCs all lived in Shanghai or the surrounding area. For both patients and HCs, we only included participants ranging in age from 18 to 65 years. Exclusion criteria for patients were as follows: suspected or diagnosed intellectual disability and presence of lifetime neurologic disease/brain trauma, hypothyroidism/hyperthyroidism, or any other clinical conditions that may influence the validity of neuropsychological assessment. Additionally, patients were included only if diagnosed with OCD while having no comorbid psychotic disorder (e.g., schizophrenia) and no major physical comorbidities. The presence of a comorbid anxiety or mood disorder did not constitute an exclusion criterion. One patient was found to have comorbid schizophrenia and was excluded from the study. HCs were included only if they reported to have no lifetime history of psychiatric disorders. They were further screened for symptoms of depression, using the Beck Depression Inventory (BDI), and those with a BDI score of more than 19 were excluded (two participants). Thus, 46 patients with OCD and $45 \mathrm{HCs}$ were included in this study. Figure 1 illustrates the exclusion, inclusion, and classification of study participants. The study was performed in accordance with the Declaration of Helsinki and approved by the ethics committees of Ruijin Hospital affiliated with Shanghai Jiao Tong University School of Medicine and Zhongshan Hospital affiliated with Fudan University. All participants provided written informed consent.

\section{Clinical Symptom Assessment}

The severity of the patients' OCD symptoms was assessed by expert psychiatrists/clinical psychologists who were blinded to the patients' CANTAB results while employing the Chinese version of the Yale-Brown Obsessive-Compulsive Symptom 


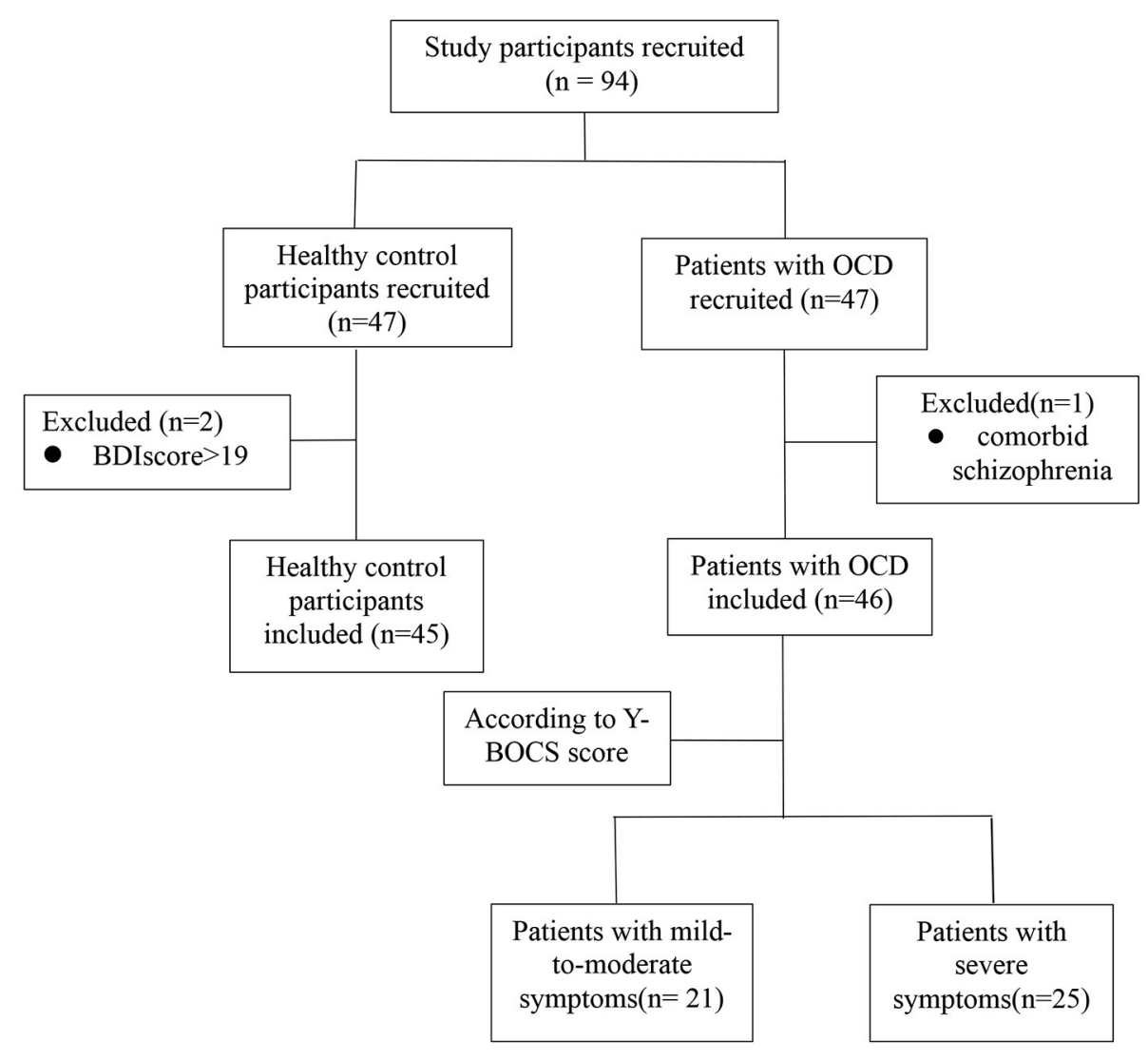

FIGURE 1 | Flowchart illustrating the exclusion, inclusion, and classification of study participants.

Checklist (Y-BOCS) (17). We used the total score on the Y-BOCS (ranging from 0 to 40 ), along with the separate subscale scores for obsessions (0-20) and compulsions (0-20), to categorize the severity of the patients' OCD symptoms as follows: "mildly severe" [total score, 6-15 $(n=4)$ or subscale scores, 6-9 for either obsessions or compulsions]; "moderately severe" [total score, $16-25(n=16)$ or subscale scores, $10-14$ for either obsessions or compulsions $(n=1)$ ]; and "severe" [total score, $>25(n=17)$, or subscale scores, 15 or higher for either obsessions $(n=8)$ or compulsions] $(18,19)$. Because the number of patients categorized as having "mildly severe" OCD symptoms was relatively low, precluding statistical analysis, we collapsed the "mildly severe" and "moderately severe" categories into one symptom category. Accordingly, the OCD group was divided into one subgroup of patients with relatively mild-to-moderate symptoms $(n=21)$ and another subgroup of patients with severe symptoms $(n=25)$. At the time of enrolment, all patients with OCD were taking selective serotonin reuptake inhibitors (SSRIs), except for four patients in the mild-to-moderate group and six patients in the severe group, who were taking no medication.

\section{Neuropsychological Assessment}

The CANTAB (CANTAB Connect Research) was administered to each participant in a quiet hospital room by a psychologist who had received intensive training in its administration.
Participants had to indicate their responses to the information in the computerized cognitive tests by touching a screen (iPad $6 \mathrm{MRJN} 2 \mathrm{CH} / \mathrm{A}$, Apple, CA, USA). We focused on testing the domain of executive functioning, in particular response inhibition, spatial working memory, planning, and attentional set shifting, along with testing new associative learning and visual recognition memory. Because not all participants were able to proceed to the next stage in each test, the number of participants yielding data for statistical analysis differed by test (Tables 1A,B).

\section{Stop Signal Task (SST)}

The SST is a choice reaction-time task purported to assess response inhibition (20). In this task, participants were required to respond (using their indexes fingers or thumbs) to an arrow ("go" signal) presented on the screen, which pointed to either the left or right. They were instructed to touch, as quickly as possible, the left side of the screen when the arrow pointed to the left and to press the right side when the arrow pointed to the right. They completed one block of 16 practice trials. Subsequently, participants performed the same task except that they had to withhold their behavioral response when an auditory ("stop") signal (a beep) was presented. The auditory stop signal was delivered at variable intervals (referred to as the stop-signal delay; SSD) after the presentation of the arrow. The stop-signal RT (SSRT), mean RT on go trials, the mean number of direction 
TABLE 1A | Number of study participants with incomplete performance data as a function of group and cognitive test.

\begin{tabular}{lcccc}
\hline Test & \multicolumn{2}{c}{ Number of Patients } & & Number of HCs \\
\cline { 2 - 4 } & \multicolumn{2}{c}{ Incomplete test data } & $\begin{array}{c}\text { Included in } \\
\text { analyses }\end{array}$ & $\begin{array}{c}\text { Incomplete test data } \\
\text { Included in } \\
\text { analyses }\end{array}$ \\
\hline SST & 3 & 43 & 0 & 4 \\
SWM & 0 & 46 & 0 & 41 \\
PAL & 0 & 46 & 11 & 45 \\
SOC & 2 & 44 & 34 \\
IED & 2 & 44 & 34
\end{tabular}

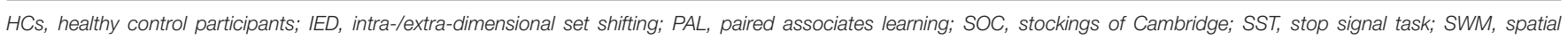
working memory.

TABLE 1B | Number of patients with incomplete performance data as a function of symptom severity.

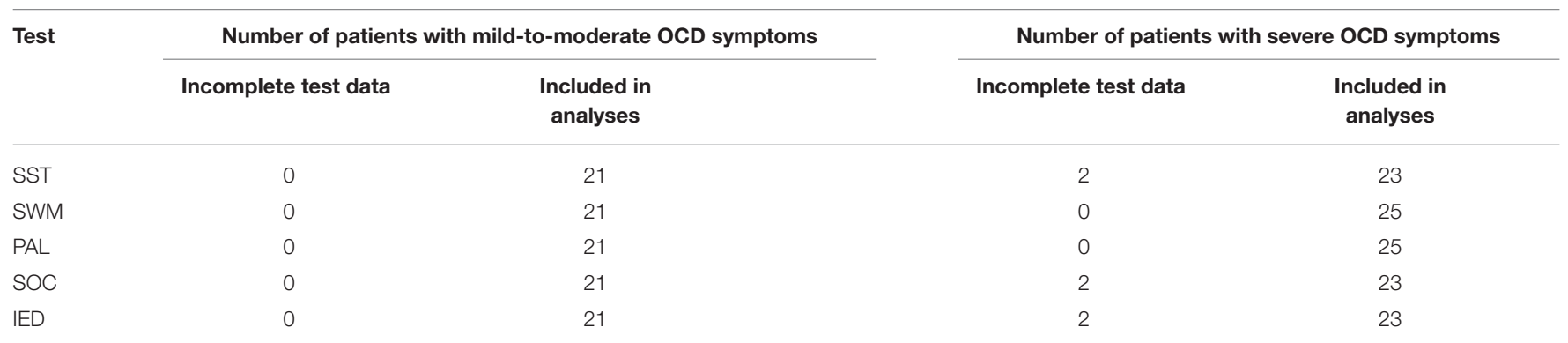

OCD, obsessive-compulsive disorder; IED, intra-/extra-dimensional set shifting; PAL, paired associates learning; SOC, stockings of Cambridge; SST, stop signal task; SWM, spatial working memory.

errors on go and stop trials, and the SSD time) served as the dependent variables.

\section{Spatial Working Memory (SWM)}

The SWM task measures the capacity to retain and manipulate spatial information for performing the task at hand. In this task, participants were presented with multiple boxes in an increasing order on the screen, with each box revealing a token after being tapped on. All tokens were dropped in a column, and participants were instructed to avoid the box where they had previously found a token. The main dependent variable was the total number of errors made by participants, that is, the errors associated with returning to the box where a token was previously found (21).

\section{Paired Associates Learning (PAL)}

The PAL test, involving new associative learning and visual recognition memory, required participants to recall a location previously paired with an object. In this task, they were presented with a set of boxes on the screen, which automatically opened and revealed an object/pattern. The patterns emerging from the boxes during the task were different and occurred one at a time in a randomized order. Subsequently, each of the patterns was displayed one at a time on the center of the screen, and participants were asked to identify the box previously associated with the pattern. The dependent variables consisted of the total number of patterns reached, the total number of attempts, and the total errors adjusted.

\section{Stockings of Cambridge (SOC)}

The SOC test evaluates planning, that is, the ability to cognitively select an adequate action to reach a desired goal. The participants were shown two images stacked rowwise, where the top image had three stockings suspending three colored balls. The participants were instructed to move the balls in the bottom image in order to replicate the top pattern. The balls could be moved only one at a time and were accompanied by a maximum number of allowed moves. The dependent variables were the number of SOC problems that participants successfully completed in the minimum possible number of moves, and the mean number of moves they required to complete 5-move SOC problems.

\section{Intra-Extra Dimensional Set Shift (IED)}

The IED test assesses rule acquisition and reversal involving visual discrimination and attentional set shifting. In this task, participants were required to evaluate visual stimuli along one or two physical dimensions (form and color) and to use feedback in order to discover a rule that determined which stimulus was correct. After six correct responses, the rule and/or stimuli changed. Initially, participants could distinguish the visual stimuli easily on the basis of one relevant dimension and the subsequent shifts in rule were intradimensional. Next, the visual stimuli could be distinguished only on the basis of a combination of the two stimulus dimensions and the shifts in rule were extra-dimensional. 
TABLE 2A | Clinical and demographic characteristics of study participants.

\begin{tabular}{|c|c|c|c|c|c|c|c|}
\hline & & \multicolumn{2}{|c|}{ Patients(n=46) } & \multicolumn{2}{|c|}{ HCs $(n=45)$} & \multicolumn{2}{|c|}{ Between-group comparison } \\
\hline \multicolumn{2}{|c|}{ Sex (male/female) } & $25 / 21$ & & $22 / 23$ & & $Z=-0.52$ & 0.604 \\
\hline \multicolumn{2}{|c|}{ Education (years) } & $13.6(3.6)$ & $5-22$ & $14.1(3.1)$ & $5-19$ & $Z=-0.48$ & 0.634 \\
\hline \multicolumn{2}{|c|}{ Illness duration (years) } & $11.3(7.6)$ & $1-36$ & & & & \\
\hline \multirow{2}{*}{ Y-BOCS score } & Compulsion & $10.0(6.4)$ & $0-20$ & & & & \\
\hline & Total score & 23.5(8.8) & $4-38$ & & & & \\
\hline
\end{tabular}

HCs, healthy control participants; M, mean; SD, standard deviation; Y-BOCS, Yale-Brown Obsessive-Compulsive Symptom Checklist.

TABLE 2B | Clinical and demographic characteristics of patients classified according to symptom severity.

\begin{tabular}{|c|c|c|c|c|c|c|c|}
\hline & & \multicolumn{2}{|c|}{$\begin{array}{c}\text { Patients with } \\
\text { mild-to-moderate OCD } \\
\text { symptoms }(n=21)\end{array}$} & \multicolumn{2}{|c|}{$\begin{array}{c}\text { Patients with severe OCD } \\
\text { symptoms }(n=25)\end{array}$} & \multicolumn{2}{|c|}{ Between-group comparison } \\
\hline & & $M(S D)$ & Range & $M(S D)$ & Range & Test statistic & $\boldsymbol{P}$ \\
\hline Age (years) & & $34.7(9.3)$ & $21-56$ & $31(7.6)$ & $19-49$ & $t=1.47$ & 0.149 \\
\hline Sex (male/female) & & $9 / 12$ & & $16 / 9$ & & $Z=1.42$ & 0.156 \\
\hline Education (years) & & $14.3(3.5)$ & $8-22$ & $13(3.6)$ & $5-18$ & $Z=-0.91$ & 0.364 \\
\hline Illness Duration (years) & & $11.2(7.2)$ & $2-29$ & $11.4(8.1)$ & $1-36$ & $t=-0.09$ & 0.927 \\
\hline \multirow[t]{3}{*}{ Y-BOCS (subscale and scale score) } & Obsession & $9.1(3.9)$ & $0-16$ & $17.1(2.3)$ & 13-20 & $Z=-5.46$ & $<0.001$ \\
\hline & Compulsion & $8.6(3.2)$ & $0-13$ & $11.2(8)$ & $0-20$ & $Z=-2.14$ & 0.032 \\
\hline & Total score & $17.7(5.8)$ & $4-25$ & $28.3(8.1)$ & $15-38$ & $t=-4.99$ & $<0.001$ \\
\hline
\end{tabular}

OCD, obsessive-compulsive disorder; M, mean; SD, standard deviation; Y-BOCS, Yale-Brown Obsessive-Compulsive Symptom Checklist.

There were nine stages to be completed in the task, with intra- and extra-dimensional rule shifts linked to attentional set shifting occurring at stages 6 and 8 , respectively. The dependent variables comprised the number of errors made at stages 4,6 , and 8 , as well as the number of stimulus trials completed successfully.

\section{Statistical Analysis}

Initially, we evaluated whether the continuous dependent variables were normally distributed. If this requirement was met, we performed independent-sample $t$-tests to assess mean differences between the patients and HCs and, within the patient group, between patients with mild-to-moderate symptoms and patients with relatively severe symptoms. We conducted Mann-Whitney $U$ tests if the normality requirement was not met, and for analyzing differences in the proportion of males and females between groups. Because the CANTAB yielded 15 cognitive performance measures (5 from SST, 1 from SWM, 3 from PAL, 2 from SOC, 4 from IED), a Bonferroni-adjusted level of significance of 0.003 ( $p=0.05 / 15=0.003$, two-tailed) was used to protect against inflated Type I error rates (false positives) due to multiple testing. SPSS v26.0 (IBM, Armonk, NY) was used to analyze the data. The data are available upon request from the corresponding author.

\section{RESULTS}

There were no significant group differences seen between the patients with OCD and HCs in relation to age $(p=0.107)$, sex $(p=0.604)$, and education $(p=0.634)$ (Table 2A). Also, no significant differences were observed between patients with mildto-moderate symptoms and patients with severe symptoms in age $(p=0.149)$, sex $(p=0.156)$, education $(p=0.364)$, and illness duration $(p=0.947)$ (Table 2B).

Table 3 presents the performance data derived from the SST, SWM, PAL, SOC, and IED separately for patients and HCs along with the results of the statistical analysis. Table 4 summarizes the performance data obtained from the patients classified by OCD symptom severity. No significant patient-control differences were observed in any of the cognitive performance measures (all $p>0.003$ ) (Table 3). Similarly, cognitive performance of patients with relatively mild-to-moderate OCD symptoms did not differ significantly from the performance of patients with severe symptoms (all $p>0.003$ ) (Table 4).

\section{DISCUSSION AND CONCLUSION}

In this study, we employed the CANTAB to evaluate several executive functions in Chinese patients with OCD and HCs. No significant patient-control differences were observed in the 
TABLE 3 | Cognitive performance data as a function of group and test along with results of between-group analysis*.

\begin{tabular}{|c|c|c|c|c|c|}
\hline Test & Performance measure & Patients & HCs & Test statistics & $P$ \\
\hline \multirow[t]{5}{*}{ SST } & Stop Signal Reaction Time (ms) & $238.3(35.7)$ & $269.8(69.2)$ & $Z=-1.95$ & 0.052 \\
\hline & SST Median RT (ms) & $513.8(67.1)$ & $496.7(58.0)$ & $t=-1.28$ & 0.204 \\
\hline & SST Direction Errors: Go Trials & $1.6(2.6)$ & $2.1(3.2)$ & $Z=-0.67$ & 0.501 \\
\hline & SST Direction Errors: Stop Trials & $41.5(3.3)$ & $43.3(3.9)$ & $Z=-2.55$ & 0.011 \\
\hline & Stop signal delay (ms) & $256(96.5)$ & $231.5(65.2)$ & $t=-1.41$ & 0.161 \\
\hline \multirow{2}{*}{ PAL } & Total Attempts & $7.4(2.1)$ & $7.8(2.0)$ & $Z=-1.09$ & 0.274 \\
\hline & Total Errors (Adjusted) & $15.4(12.8)$ & $14.4(10.8)$ & $Z=-0.12$ & 0.902 \\
\hline \multirow[t]{2}{*}{ soc } & Problems Solved in Minimum Moves Total (all moves) & $8.2(2.3)$ & $7.4(2.3)$ & $Z=-1.43$ & 0.153 \\
\hline & Mean Moves (five Moves) & $6.9(1.7)$ & $7.2(1.6)$ & $t=-0.86$ & 0.392 \\
\hline IED & Errors (Stage 4) & $0.7(2.4)$ & $1.1(3.9)$ & $Z=-1.36$ & 0.174 \\
\hline
\end{tabular}

HCs, healthy control participants; IED, intra-/extra-dimensional set shifting; PAL, paired associates learning; SOC, stockings of Cambridge; SST= stop signal task; SWM, spatial working memory.

*Data values represent group means and standard deviations.

TABLE 4 | Performance data as a function of patient subgroup and cognitive test along with results of between-subgroup analysis*.

\begin{tabular}{|c|c|c|c|c|c|}
\hline Test & Performance measure & $\begin{array}{l}\text { Patients with } \\
\text { mild-to-moderate OCD } \\
\text { symptoms }\end{array}$ & $\begin{array}{l}\text { Patients with severe OCD } \\
\text { symptoms }\end{array}$ & Test statistic & $\boldsymbol{P}$ \\
\hline \multirow{3}{*}{ SST } & SST Median RT (ms) & $524.9(66.6)$ & $503.3(67.4)$ & $t=1.06$ & 0.296 \\
\hline & SST Direction Errors: Go Trials & $0.9(1.6)$ & $2.3(3.2)$ & $Z=-1.55$ & 0.121 \\
\hline & SST Direction Errors: Stop Trials & $40.7(3.2)$ & $42.3(3.3)$ & $t=-1.62$ & 0.113 \\
\hline SWM & Total Errors & $9.3(7.6)$ & $7.9(8.2)$ & $Z=-0.72$ & 0.469 \\
\hline \multirow[t]{3}{*}{ PAL } & Number of Patterns Reached & $7.8(0.6)$ & $7.8(0.7)$ & $Z=-0.27$ & 0.790 \\
\hline & Total Attempts & $8.0(2.4)$ & $6.9(1.7)$ & $Z=-1.43$ & 0.153 \\
\hline & Total Errors (Adjusted) & $17.5(14.0)$ & $13.7(11.6)$ & $t=-0.99$ & 0.326 \\
\hline SOC & Problems Solved in Minimum Moves Total (all moves) & $7.9(2.7)$ & $8.5(2.0)$ & $t=-0.87$ & 0.387 \\
\hline & Stages Completed & $8.1(1.3)$ & $8.3(1.7)$ & $Z=-1.05$ & 0.292 \\
\hline
\end{tabular}

OCD, obsessive-compulsive disorder; IED, intra-/extra-dimensional set shifting; PAL, paired associates learning; SOC, stockings of Cambridge; SST = stop signal task; SWM, spatial working memory.

*Data values represent group means and standard deviations.

performance of tests of response inhibition, spatial working memory, planning, and set shifting. In addition, the two groups displayed no significant differences in cognitive performance involving basic learning and memory. Moreover, within the patient group, no significant performance differences were detected between patients who were classified as having either relatively mild or severe OCD symptoms. These results are unexpected and do not seem to support the view that impaired executive functioning is a core cognitive and pathophysiological feature of OCD (5-9). However, several factors partly related to limitations of the present study need to be considered before this conclusion can be well accepted.

First, our patient group was comparable to the HC group with respect to age, sex, and education, but it remains possible that preexisting group differences in other variables relevant to cognitive performance, such as socioeconomic status, medication 
status, or intelligence, contributed to the present results. For example, our OCD group mainly consisted of patients who were taking SSRIs at the time of testing, which may have improved their cognitive performance (22). Yet, medication status cannot easily explain the nonsignificant differences between patients with relatively mild and severe OCD symptoms because most patients in both subgroups were taking SSRIs at the time of testing.

Second, although the present study was not a crosscultural study and the CANTAB is presumed to be culturally independent, it is possible that cultural factors contributed to the present results, precluding a direct comparison with prior findings from studies conducted in Western societies and patient populations. Therefore, it remains to be determined whether the present findings can be generalized to patients with OCD in other cultures and societies. Third, we employed the Bonferroni correction, which is an adequate but conservative method for controlling Type I errors (false positive findings) due to multiple testing. Accordingly, the use of this method may have controlled Type I errors but at the cost of increasing Type II errors (false negatives) and hence, may have reduced the statistical power of the study to detect small but true patient-control differences in cognitive performance. Indeed, meta-analytical reviews of the literature indicate that patient-control differences in cognitive performance are generally modest, with effect sizes ranging from small to medium for tests of EF $(12,13)$. Similarly, within a given cognitive task, effect sizes may differ across the dependent variables used for analyzing performance differences [e.g., for SST, a large effect size has been found for SSRT but only a small and nonsignificant effect for performance accuracy (14)].

However, due to some limitations of this study, these results should be interpreted with caution. The first limitation of the present study concerns the small sample sizes examined, which seem to be insufficient to reliably detect cognitive deficits in patients with OCD. Secondly, influenced by the sample size, we did not make a detailed division according to the symptoms of OCD for cognitive comparison. Thirdly, more research and cross-cultural studies are needed to determine whether these results can be replicated in another sample of Chinese patients and whether they can truly be generalized to patients living in other countries and sociocultural cultures.

In conclusion, we observed no significant differences between Chinese patients with OCD and healthy community volunteers in cognitive tests assessing executive functions. However, due to study limitations, additional cognitive studies including large, well-characterized samples of Chinese patients with OCD and

\section{REFERENCES}

1. Goodman WK, Grice DE, Lapidus KA, Coffey BJ. Obsessivecompulsive disorder. Psychiatr Clin North Am. (2014) 37:25767. doi: 10.1016/j.psc.2014.06.004

2. Torres AR, Prince MJ, Bebbington PE, Noskova E, Koprivova J, Stopkova P, et al. Obsessive-compulsive disorder: prevalence, comorbidity, impact, and help-seeking in the British National Psychiatric Morbidity Survey of 2000. Am J Psychiatry. (2006) 163:1978-85. doi: 10.1176/ajp.2006.163.11.1978 matched HCs, as well as cross-cultural studies, are needed to substantiate or qualify the present findings.

\section{DATA AVAILABILITY STATEMENT}

The raw data supporting the conclusions of this article will be made available by the authors, without undue reservation.

\section{ETHICS STATEMENT}

The studies involving human participants were reviewed and approved by Ruijin Hospital and Zhongshan Hospital. The patients/participants provided their written informed consent to participate in this study.

\section{AUTHOR CONTRIBUTIONS}

ZZ, WL, and CZ were responsible for the study concept and design. HL and RC contributed to the acquisition of behavioral data. JH and NZ assisted with data analysis and interpretation of findings. $\mathrm{HR}$ and $\mathrm{JH}$ drafted the manuscript. $\mathrm{ZZ}$ and $\mathrm{WL}$ provided critical revision of the manuscript for important intellectual content. All authors critically reviewed content and approved the final version for publication.

\section{FUNDING}

Natural Science Foundation of China Grant (81771482). The Second Batch of Novel Coronavirus Prevention and Control Emergency Science and Technology project in Xinxiang City (20GG007).

\section{ACKNOWLEDGMENTS}

The authors would like to thank our colleagues at Ruijin Hospital affiliated to Shanghai Jiao Tong University School of Medicine and Zhongshan Hospital affiliated to Fudan University who help immensely with recruitment. The authors would also like to thank all study participants.

\section{SUPPLEMENTARY MATERIAL}

The Supplementary Material for this article can be found online at: https://www.frontiersin.org/articles/10.3389/fpsyt. 2021.662449/full\#supplementary-material 
6. Yazdi-Ravandi S, Shamsaei F, Matinnia N, Moghimbeigi A, Shams J, Ahmadpanah $\mathrm{M}$, et al. Executive functions, selective attention and information processing in patients with obsessive compulsive disorder: A study from west of Iran. Asian J Psychiatr. (2018) 37:140-5. doi: 10.1016/j.ajp.2018.09.002

7. Martoni RM, de Filippis R, Cammino S, Giuliani M, Risso G, Cavallini MC, Bellodi L, et al. Planning functioning and impulsiveness in obsessivecompulsive disorder. Eur Arch Psychiatry Clin Neurosci. (2018) 268:47181. doi: 10.1007/s00406-017-0803-0

8. Menzies L, Chamberlain SR, Laird AR, Thelen SM, Sahakian BJ, Bullmore ET. Integrating evidence from neuroimaging and neuropsychological studies of obsessive-compulsive disorder: the orbitofronto-striatal model revisited. Neurosci Biobehav Rev. (2008) 32:525-49. doi: 10.1016/j.neubiorev.2007.09.005

9. Fajnerova I, Gregus D, Francova A, Noskova E, Koprivova J, Stopkova $\mathrm{P}$, et al. Functional connectivity changes in obsessive-compulsive disorder correspond to interference control and obsessions severity. Front Neurol. (2020) 11:568. doi: 10.3389/fneur.2020.00568

10. Menzies L, Williams GB, Chamberlain SR, et al. White matter abnormalities in patients with obsessive-compulsive disorder and their first-degree relatives. Am J Psychiatry. (2008) 165:1308-15. doi: 10.1176/appi.ajp.2008.07101677

11. Miyake A, Friedman NP. The nature and organization of iIndividual differences in executive functions: four general conclusions. Curr Dir Psychol Sci. (2012) 21:8-14. doi: 10.1177/0963721411429458

12. Snyder HR, Kaiser RH, Warren SL, Heller W. Obsessive-compulsive disorder is associated with broad impairments in executive function: A meta-analysis. Clin Psychol Sci. (2015) 3:301-30. doi: 10.1177/2167702614534210

13. Shin NY, Lee TY, Kim E, Kwon JS. Cognitive functioning in obsessivecompulsive disorder: a meta-analysis. Psychol Med. (2014) 44:112130. doi: 10.1017/S0033291713001803

14. Abramovitch A, Abramowitz JS, Mittelman A. The neuropsychology of adult obsessive-compulsive disorder: a meta-analysis. Clin Psychol Rev. (2013) 33:1163-71. doi: 10.1016/j.cpr.2013. 09.004

15. BakerE, Dente P, BackxR, Lowther M, Cotter J, CormackF.K, et al. Delivery of CANTAB assessments across diverse health systems: developing topics. Alzheimer's \& Dementia. (2020) 16. doi: 10.1002/alz.0 47550

16. Fray PJ, Robbins TW, CANTAB. battery: proposed utility in neurotoxicology. Neurotoxicol Teratol. (1996) 18:499-504. doi: 10.1016/0892-0362(96)0 0027-X
17. Storch EA, Shapira NA, Dimoulas E, Geffken GR, Murphy TK, Goodman WK Yale-Brown Obsessive Compulsive Scale: the dimensional structure revisited. Depress Anxiety. (2005) 22:28-35. doi: 10.1002/da.20088

18. Goodman WK, Price LH, Rasmussen SA, Mazure C, Fleischmann RL, Hill CL, et al. The yale-brown obsessive compulsive scale I. Development, use, and reliability. Arch Gen Psychiatry. (1989) 46:100611. doi: 10.1001/archpsyc.1989.01810110048007

19. Goodman WK, Price LH. Assessment of severity and change in obsessive compulsive disorder. Psychiatr Clin North Am. (1992) 15:861-9. doi: 10.1016/S0193-953X(18)30214-4

20. Logan GD, Cowan WB, Davis KA. On the ability to inhibit simple and choice reaction time responses: a model and a method. J Exp Psychol Hum Percept Perform. (1984) 10:276-91. doi: 10.1037/0096-1523.10.2.276

21. Owen AM, Downes JJ, Sahakian BJ, Polkey CE, Robbins TW. Planning and spatial working memory following frontal lobe lesions in man. Neuropsychologia. (1990) 28:1021-34. doi: 10.1016/0028-3932(90)9 0137-D

22. Skandali N, Rowe JB, Voon V, Deakin JB, Cardinal RN, Cormack F, et al. Dissociable effects of acute SSRI (escitalopram) on executive, learning and emotional functions in healthy humans. Neuropsychopharmacology. (2018) 43:2645-51. doi: 10.1038/s41386-0180229-z

Conflict of Interest: The authors declare that the research was conducted in the absence of any commercial or financial relationships that could be construed as a potential conflict of interest.

Publisher's Note: All claims expressed in this article are solely those of the authors and do not necessarily represent those of their affiliated organizations, or those of the publisher, the editors and the reviewers. Any product that may be evaluated in this article, or claim that may be made by its manufacturer, is not guaranteed or endorsed by the publisher.

Copyright (C) 2021 Ren, Li, Huang, Zhang, Chen, Liu, Zhang and Zhang. This is an open-access article distributed under the terms of the Creative Commons Attribution License (CC BY). The use, distribution or reproduction in other forums is permitted, provided the original author(s) and the copyright owner(s) are credited and that the original publication in this journal is cited, in accordance with accepted academic practice. No use, distribution or reproduction is permitted which does not comply with these terms. 